\title{
Le syndrome de Pierre Robin et le dysfonctionnement néonatal du tronc cérébral
}

\section{RÉSUMÉ}

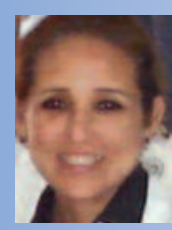

Hanane BENHALIMA

Professeur agrégé en stomatologie et chirurgie maxillo-faciale, Hôpital des spécialités, C.H.U. de Rabat,

Quartier Souissi,

10000 - Rabat,

Maroc.

\section{Misk MÉZIANE}

Résidente en stomatologie et chirurgie maxillo-faciale, Hôpital des spécialités,

C.H.U. de Rabat,

Maroc

\section{Mohamed BOULAICH}

Chef du service ORL

et chirurgie maxillo-faciale, Hôpital des spécialités,

C.H.U. de Rabat,

Maroc

Mohamed KZADRI

Chef du service ORL et chirurgie maxillo-faciale,

Hôpital des spécialités,

C.H.U. de Rabat,

Maroc

Le syndrome de Pierre Robin (SPR) touche un enfant sur 8500 par an en France. L'étiopathogénie est encore obscure. Toutefois, la théorie actuellement retenue est celle d'un dysfonctionnement néonatal du tronc cérébral (ou rhombencéphale), responsable des signes malformatifs et des troubles oro-faciaux qui peuvent être dramatiques. Le SPR constitue une urgence néonatale qui doit être bien prise en charge par une équipe spécialisée multidisciplinaire.

syndrome de Pierre Robin

- rhombencéphale

urgence néonatale
AOS 2011;254:163-170

DOI: $10.1051 /$ aos/2011208

(C) AEOS / EDP Sciences 


\section{Introduction}

$>$

Le syndrome de Pierre Robin (SPR) se définit par l'association d'une fente palatine postérieure, d'un rétrognatisme et d'une glossoptose (fig. 1) [1].

Grâce aux progrès de la neurobiologie embryonnaire, l'ensemble des anomalies observées à la naissance est la conséquence d'un trouble de la neurogenèse du tronc cérébral (rhombencéphale) autour des dixième-douzième semaines de vie.

Le syndrome a été individualisé comme un dysfonctionnement néonatal du tronc cérébral responsable d'un défaut d'organisation des fonctions de la succion-déglutition, de la commande ventilatoire, de la motricité œsophagienne et d'une dysrégulation ortho-parasympathique cardiaque [1].

Les formes graves constituent souvent une urgence néonatale dont le taux de décès varie de $25 \%$ à $26 \%$ des cas pour Couly [2].

\section{Éthio-épidémiologie}

\section{Fréquence}

Environ 60 à 80 enfants sont touchés chaque année en France, soit 1 enfant sur 8500 [2]. Le SPR est isolé dans $50 \%$ des cas, dans l'autre moitié il fait partie d'un syndrome malformatif plus complexe.

\section{Étiologie}

Le mécanisme pathogénique est imprécis. Aucun gène n'est connu dans le SPR isolé. Toutefois, des facteurs tératogènes (irradiation, rubéole, syphilis, Tégrétol ${ }^{\circledR}$, phénobarbital,
La prise en charge difficile et longue de ces malades doit être réalisée par une équipe multidisciplinaire associant le chirurgien pédiatre, le réanimateur, le chirurgien maxillo-facial et l'oto-rhino-laryngologiste.

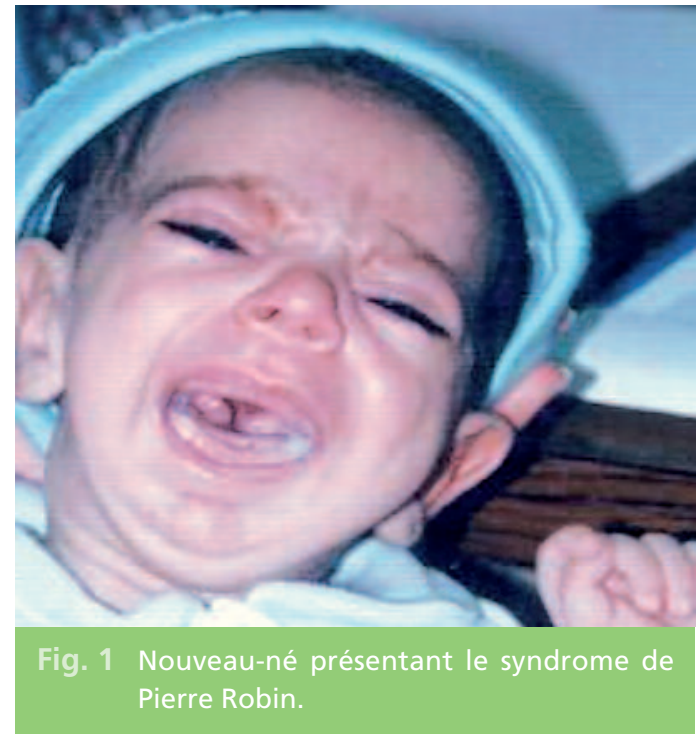

carence en acide folique, hypovitaminose...) peuvent être incriminés [4]

\section{Conception actuelle du dysfonctionnement néonatal du tronc cérébral [2]}

Couly et al. [2] ont démontré que le SPR est l'expression d'un trouble précoce de la neurogenèse du tronc cérébral (rhombencéphale) au niveau soit du tube neural, soit des crêtes neurales rhombencéphaliques (rhombomères 6, 7 et 8). 
La neurulation ( $25^{\mathrm{e}}-50^{\mathrm{e}}$ jours) correspond à la mise en place du tube neural et à la migration des cellules des crêtes neurales vers les bourgeons faciaux dont le rôle dans l'organogenèse craniofaciale est actuellement établi (fig. 2).

Le rhombencéphale est la troisième portion du tube neural d'où naît le cervelet et le bulbe rachidien. À ce niveau également, naissent les huit dernières paires crâniennes dont six (V, VII, IX, X, XI, XII) participent à l'innervation motrice, sensorielle et sensitive des régions faciale, buccale et cervicale. On retrouve aussi les noyaux des nerfs moteurs oculaires externes (VI), de l'audition, de I'équilibre (VIII), les noyaux cardiopneumo-entériques, les centres respiratoires et la substance réticulée bulbo pontique (fig. 3).

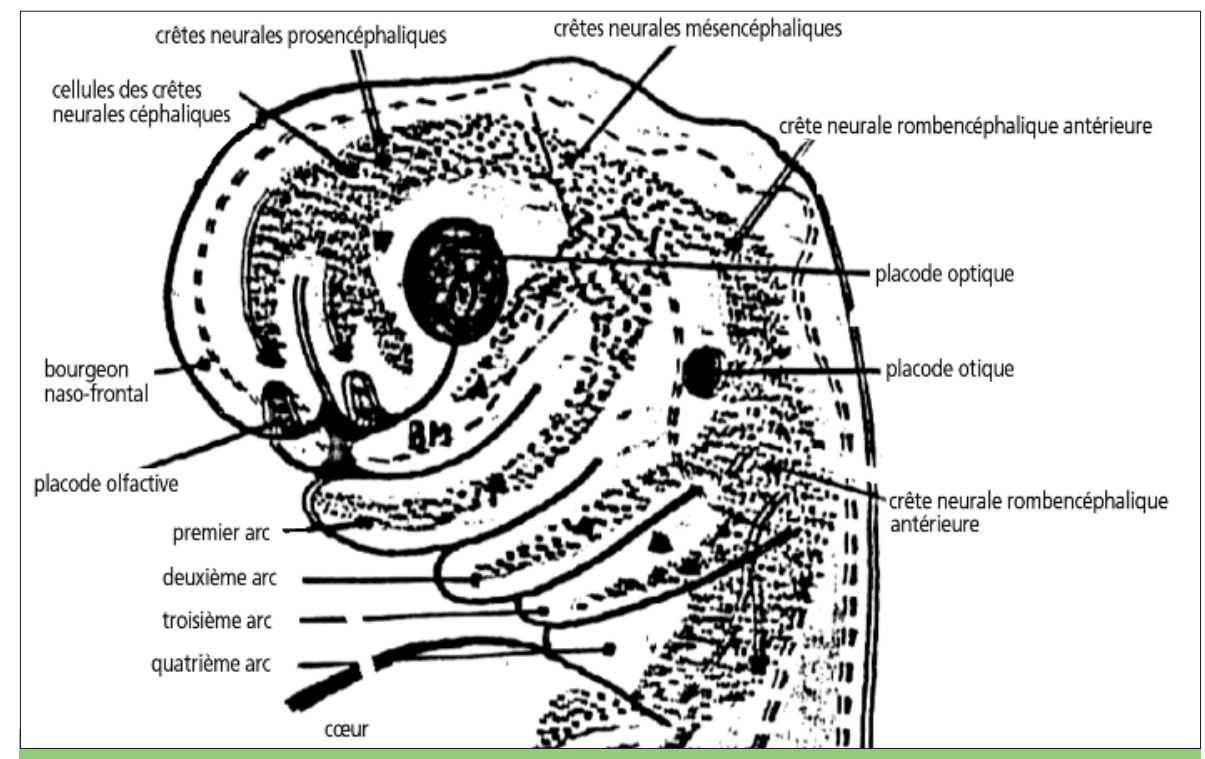

Fig. 2 Schéma de l'extrémité céphalique de l'embryon au bout de deuxième mois.

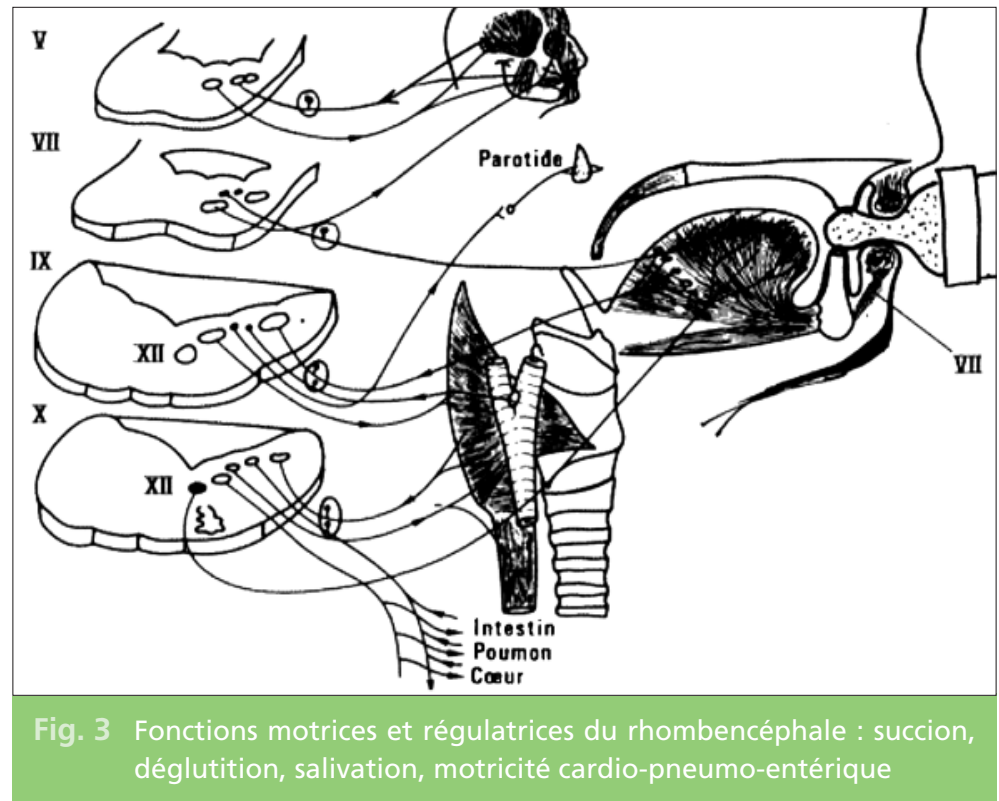


Le défaut de la matrice fœtale de la langue gène sa descente de la base du crâne vers la cavité buccale ( 9 e semaine) et entrave la fusion des procès palatins provoquant une fente vélo-palatine. La non-acquisition de la succion-déglutition ( $10^{\mathrm{e}}-12^{\mathrm{e}}$ semaine) est responsable de la glossoptose et de la rétrognathie $[2,3,5,6]$.

\section{Diagnostic positif du SPR}

Le diagnostic du syndrome de Pierre Robin est essentiellement clinique, il doit être évoqué chez tout nouveau-né présentant, plus ou moins associés, la triade malformative faciale et des troubles des fonctions orofaciale.

\section{La triade malformative faciale}

Rétro-mandibulie avec aspect caractéristique en " tête d'oiseau ", glossoptose et fente palatine ou vélo-palatine qui n'apparaît pas constante (70 à $83 \%$ des cas pour Delmotte).

\section{Les troubles}

des fonctions orofaciales

\section{$>$ Troubles de succion-déglutition}

Ils sont marqués par des tétées inefficaces et par des fausses routes alimentaires. La fibroscopie et particulièrement la vidéoscopie permettent d'évaluer la motricité oro-pharyngolaryngée. Elle a une tolérance bonne de la naissance à 18 mois puis sans grandes difficultés à partir de 3 ans $[7,8]$.

\section{$>$ Troubles respiratoires}

C'est la principale cause de mortalité chez $25 \%$ des nourrissons selon Couly. On distingue :

- les apnées centrales dues à un arrêt de la commande respiratoire ;
- les apnées obstructives dues à l'obstruction rétro-basi-linguale des voies aériennes supérieures ;

- le syndrome d'apnées du sommeil (SAOS) qui correspond à plus de dix pauses respiratoires supérieures à 10 secondes par heure ou de 15 apnées hypoxies par heure du sommeil. La polysomnographie, l'oxymétrie nocturne (SAO2) et l'exploration fonctionnelle respiratoire sont indispensables pour quantifier leur gravité $[9,10]$.

\section{> Motricité œsophagienne}

À type de reflux gastro-œsophagien et une dysphagie présents chez $94 \%$ des nourrissons.

La manométrie est l'examen essentiel pour objectiver un éventuel asynchronisme pharyngo-laryngé, un trouble de péristaltisme, des anomalies de sphincters ou au maximum une achalasie œsophagienne [10].

\section{> Dysrégulation cardiaque}

À type d'accès de pâleur ou de cyanose voire des syncopes (59\% des cas). Le diagnostic est confirmé par l'électrocardiogramme couplé au réflexe oculo-cardiaque.

Il y a une hyperréactivité vagale si l'asystolie provoquée par la compression oculaire est supérieure à $4000 \mathrm{~ms}[9,10]$. 


\section{Classification clinique (Couly, 1998)}

- Le stade I (25\% des cas) correspond aux critères malformatifs suscités sans troubles oraux ou ventilatoires.

- Le stade II (40 \% des cas) comporte des troubles modérés mais susceptibles de s'aggraver à tout moment.
- Le stade III (15\% des cas) associe des pauses ventilatoires graves aux troubles de l'oralité qui peuvent être dramatiques. Il pose de sérieuses difficultés de prise en charge thérapeutique [11].

\section{Aspects évolutifs du SPR}

L'évolution propre de la séquence de Pierre Robin est de pronostic favorable. Le rétrognatisme s'atténue ou disparaît vers l'âge de 2-3 ans et la langue reprend sa position normale.

Cependant, des complications, plus ou moins graves, peuvent survenir. Nous citons :

- les broncho-pneumopathies chroniques qui sont secondaires aux fausses routes alimentaires et au reflux gastro-œsophagien ;

- l'hyperpression artérielle pulmonaire dont le risque ultime est le cœur pulmonaire chronique ;

- I'encéphalopathie hypoxémique qui est à l'origine du retard psychomoteur et de la mauvaise acquisition des fonctions cognitives ;
- I'insuffisance vélaire, ou rhinolalie ouverte, responsable de nasonnement par fuite d'air et des bruits de remplacement ;

- une surdité de transmission et des otites séro-muqueuses qui sont plus fréquentes dans le SPR par opposition aux fentes palatines isolées (73\% versus $58 \%$ [12]) dus à l'inflammation du nasopharynx et au dysfonctionnement tubaire. Elles doivent être dépistées dès les premiers mois de vie par des potentiels évoqués auditifs, des examens otoscopiques réguliers et des impédancemétries ;

- le retard de croissance staturo-pondérale dû à l'acidose tissulaire, qui semble entraver la sensibilité à l'hormone de croissance [13, 14].

\section{Prise en charge thérapeutique}

Parmi les objectifs premiers du traitement du SPR, citons :

- La levée de l'obstruction glosso-pharyngolaryngée dont les moyens varient selon la gravité :

- l'orthostatisme ventral dans les formes mineures ;
- les glossopexies qui réalisent un accolement de la langue à la gencive ou à la lèvre ;

- I'hypomandibulopexie qui consiste à rapprocher l'os hyoïde du bord inférieur de la mandibule. Plus récemment, la technique de désinsertion sous-périostée des muscles $d u$ plancher buccal semble la plus retenue ; 
- I'intubation endotrachéale ou trachéotomie salvatrice d'une mort certaine $[15,16]$.

\section{- La correction des troubles alimentaires et} du reflux œsophagien peut être obtenue par :

- le nursing, le gavage gastrique ou la gastrostomie ;

- la prescription médicamenteuse anti-reflux avec épaississement des repas ;

- en cas d'échec, le recours à l'intervention de
Nissen par manchonnement péri-œsophagien de la grosse tubérosité gastrique.

- La fermeture de la fente palatine, par myoplastie intra-vélaire entre 3 et 18 mois, pour assurer un développement dento-maxillaire et phonétique correct [17].

\section{- La correction de la rétro-mandibulie}

Grâce à la distraction osseuse précoce de la mandibule sans attendre la fin de la croissance pour proposer une chirurgie orthognatique ou les ostéotomies [18].

\section{Conclusion}

Le SPR constitue une incontestable urgence néonatale dont l'évolution peut être dramatique. La théorie actuelle du dysfonctionnement néonatal du tronc cérébral semble être à l'origine des divers dysfonctionnements oro-faciaux et ventilatoires observés chez le nouveau-né.

L'échographie anténatale permet, actuellement, une consultation prénatale des parents pour les informer sur la malformation, sur les difficultés et les différentes étapes du traitement. Le conseil génétique est peu requis pour les SPR isolés. En revanche, il est indispensable dans les syndromes malformatifs si l'enquête familiale est négative.

Une équipe multidisciplinaire s'impose, associant le chirurgien pédiatre, le réanimateur, le chirurgien maxillo-facial, l'orthophoniste, l'oto-rhinolaryngologiste et bien d'autres spécialités intégrées en fonction des troubles ou des complications observés, pour une meilleure prise en charge du nouveau-né de la consultation prénatale jusqu'à la fin de la croissance.

\section{Bibliographie}

1. Robin P.

La chute de la base de la langue considérée comme une nouvelle cause de gène dans la respiration nasopharyngienne. Bull Acad Med 1923;89:37-40.

2. Couly G. Nouvelle conception de la maladie et du syndrome de Pierre Robin : dysneurulation du rhombencéphale.
Rev Stomatol Chir Maxillofac 1983;84(4):225-32.

3. Couly G, Cotley P, Cheron G, Abadie V, Martelli $\mathrm{H}$, Le Douarin NM. Rhombomères, code Hox, crêtes neurales et malformations de la face. Méd Sci 1994;10(2):151-62.

4. Marques IL, Barbieri MA, Bettiol $\mathrm{H}$.

Etiopathogenesis of isolated Robin sequence.
Cleft Palate Craniofac J 1998;35(6):517-25.

5. Hubert Ph, Manach $Y$, Cheron G, Hermabessière $C$, Cloup M. Le syndrome de Pierre Robin. Rev Prat 1991;41(1):33-7.

6. Abadie V, Campagnat J, Fortin G.

Succion, déglutition, ventilation et gènes du développement du tronc cérébral. Arch Fr Pediatr 1999;6:1043-7. 
7. Abadie V,

Morisseau-Durand MP,

Beyler C, Manach Y, Couly G.

Brainstem dysfunction:

a possible neuroembryologic pathogenesis of isolated Pierre Robin sequence.

Eur J Pediatr

2002;161(5):275-80.

8. Baudon JJ, Renault F, Goutet JM, Flores-Guevara R, Soupre V, Gold F,

Vazquez MP.

Motor dysfunction of the uper digestif tract in Pierre Robin sequence as assessed by sucking-swallowing electromyography and oesophageal manometry. J Pediatr 2002;14(6):719-23.

9. Abadie V, Cheron G, Couly G. Syndrome néonatal du tronc cérébral. Archi Fr Pediatr 1993;50:347-52.

10. Abadie V, Couly G, Manach Y. Urgence et chirurgie réparatrice en période néonatale dans le domaine cervico-maxillo-facial. Journées parisiennes de pédiatrie 2002.

Paris : Flammarion

Médecine Sciences:291-7.

11. Couly G, Cheron G, Blic J, Despres C, Cloup M, Hubert PH.

Le syndrome de Pierre Robin : classification et nouvelle approche thérapeutique. Arch Fr Pediatr 1998;43:553-9.

12. Riberti $C$, Baldo $D$, Tirone $L$, Collini M.

Hearing changes in patients with cleft lip and palate: clinico-statistical contribution. Pediatr Med Chir 1984;6(1):131-6.

13. Daskalogiannakis J, Ross RB, Tompson BD.

The mandibular catch-up growth controversy in Pierre Robin sequence. Am J Orthod Dentofac Orthop 2001;120:280-5.

14. Figueroa $A A$, Glupker $T$, Fitz MG, BeGole EA. Mandibular, tongue and airway in Pierre Robin sequence: a longitudinal cephalometric study. Cleft Palate Craniofac J
1991;28(4):425-34.

15. Médard C, Francois $M$, Narcy P.

Audition et séquence de Robin.

Ann Otolaryngol Chir Cervicofac 1999;116(6):317-21.

16. Caouette-Laberge $L$, Plamondon C, Larocque Y. Subperiosteal release of the floor of the mouth in Pierre Robin sequence. Experience with 12 cases Cleft Palate Craniofac J 1996;33:468-72.

17. Lehman JA, Fishman JRA, Neiman GS.

Treatment of the cleft palate associated with Robin sequence: Appraisal of risk factors. Cleft Palate Craniofac J 1992;32(1):25-9.

18. Monasterio FO, Drucker $\mathrm{M}$, Molina F, Ysunza A. Distraction oesteogenesis in Pierre Robin sequence and related respiratory problems in children. J Craniofac Surg 2002;13(1):79-83.

\section{SUMMARY}

\section{Pierre Robin syndrome and neonatal dysfunction of rhombencephal}

Hanane BENHALIMA, Misk MÉZIANE,

Mohamed BOULAICH, Mohamed KZADRIS

\section{Keywords \\ - Pierre Robin syndrome \\ - rhombencephal \\ - neonatal emergency}

Pierre Robin syndrome interests a new born out of 8500 per year in France. The etiopathogenesis remains still obscure and it is probably multifactorial. However, the recent theory currently selected is that of brainstem neonatal dysfunction to an early embryonic disorder of the rhombencephal neurogenesis. This dysfunction is responsible for the morphological signs and the orofacial disorders which could be dramatical. Pierre Robin syndrome is a neonatal emergency which must be well treated by a specialized multidisciplinary team. 


\section{Le syndrome de Pierre Robin \\ et le dysfonctionnement néonatal du tronc cérébral}

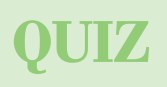

1 - Parmi les signes cliniques du syndrome de Pierre Robin( SPR) on retrouve:
a- une fente palatine
b- un rétrognatisme
c- une macroglossie
d- une rétromaxillie
e- une glossoptose

2 - Le SPR est la conséquence d'un trouble de la neurogenèse du tronc cérébral (rhombencéphale) autour de :
a- la huitième-dixième semaine de vie
b- la dixième-douzième semaine de vie
c- la douzième-quatorzième semaine de vie

3 - La prise en charge thérapeutique fait appel à :
a- la chirurgie orthognatique première
b- la trachéotomie dans les formes graves
c- la fermeture de la fente palatine postérieure
d- la gastrostomie d'alimentation

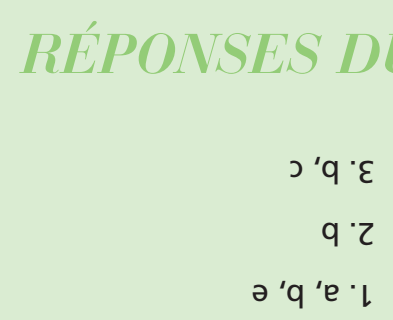

